\title{
Avaliação da integralidade na atenção primária à saúde através da Primary Care Assessment Tool: revisão sistemática
}

\author{
Julia da Rosa Tolazzi, ${ }^{1}$ Gabriela Monteiro Grendene ${ }^{2}$ e Daniele Botelho Vinholes ${ }^{1}$
}

Como citar

Tolazzi JR, Grendene GM, Vinholes DB. Avaliação da integralidade na atenção primária à saúde através da Primary Care Assessment Tool: revisão sistemática. Rev Panam Salud Publica. 2022;46:e2. https://doi.org/10.26633/RPSP.2022.2

RESUMO

Objetivo. Avaliar a integralidade na atenção primária à saúde (APS) em diferentes países.

Métodos. Foi realizada uma revisão sistemática nas bases de dados PubMed, Biblioteca Virtual em Saúde e Scopus. Foram selecionados estudos observacionais publicados no período de 2017 a 2019, sem restrições de idioma ou país, que avaliaram a integralidade na APS através da Primary Care Assessment Tool (PCATool) versão adultos. A qualidade dos estudos foi avaliada por meio da escala de Newcastle-Ottawa (NOS).

Resultados. Dos 124 artigos inicialmente selecionados, 13 foram incluídos neste estudo: quatro da China, dois do Japão e quatro do Vietnã; em relação ao continente americano, todos os quatro estudos incluídos foram realizados no Brasil. Apenas um estudo da África, realizado no Malawi, foi incluído. A qualidade dos estudos conforme a NOS foi satisfatória. A avaliação da integralidade quanto à disponibilidade dos serviços classificou oito locais como tendo baixo grau de orientação à APS, enquanto cinco apresentaram alto grau de orientação. Já para os serviços prestados, nove locais foram classificados como de baixo grau de orientação, e apenas quatro apresentaram alto grau de orientação à APS.

Conclusão. O baixo grau de orientação à APS quanto à integralidade dos serviços disponíveis e prestados pode ser interpretado como a não compreensão das reais demandas dos usuários, exigindo uma ação concreta para fortalecimento da APS como base dos sistemas de saúde.

Palavras-chave Atenção primária à saúde; mecanismos de avaliação da assistência à saúde; integralidade em saúde.

A atenção primária à saúde (APS) é considerada o primeiro nível de assistência dentro das redes que compõem os serviços de saúde. Tem como características principais ser a porta de entrada dos usuários no sistema de saúde e a coordenadora da rede de atenção. Na APS, as prioridades são a prevenção e a promoção da saúde, bem como a recuperação e a reabilitação; portanto, esse nível de atenção aborda desde os problemas mais comuns até os mais raros, com a tarefa de encaminhar os usuários a outros pontos da rede quando a resolução dos agravos e das doenças depender de uma atenção mais especializada (1).

\footnotetext{
Universidade Federal de Ciências da Saúde de Porto Alegre (UFCSPA), Pós-Graduação em Tecnologias da Informação e Gestão em Saúde, Porto Alegre (RS), Brasil. $₫$ Daniele Botelho Vinholes, dani.vinholes@gmail.com
}

Starfield (1) propôs uma caracterização da APS com quatro atributos essenciais - acesso de primeiro contato, longitudinalidade, integralidade e coordenação da atenção — e três atributos derivados — orientação familiar, orientação comunitária e competência cultural. Segundo essa pesquisadora (1), a orientação da APS para esses atributos promove melhores resultados em saúde tanto para os usuários dos serviços como para o sistema como um todo, sendo portanto um aspecto a ser monitorado através de avaliações periódicas. Entre os atributos essenciais, a integralidade, entendida como o leque dos serviços que a APS pode prestar ou disponibilizar ao usuário,

\footnotetext{
2 Universidade Federal do Rio Grande do Sul (UFRGS), Pós-Graduação em Epidemiologia, Porto Alegre (RS), Brasil.
} 
representa a essência da APS, eixo central para operacionalização dos outros atributos.

Para avaliar a presença e extensão (intensidade) dos atributos propostos por Starfield, determinando assim a orientação do serviço à APS, foi desenvolvida uma ferramenta específica, a Primary Care Assessment Tool (PCATool) (2). A PCATool, utilizada em vários países, avalia os atributos da APS e, assim, a qualidade e a efetividade desse nível de atenção, a partir de entrevistas com usuários. $\mathrm{O}$ instrumento apresenta três versões, validadas em várias línguas e contextos culturais: uma para crianças, que é respondida pelos pais, responsáveis ou cuidadores; uma para adultos maiores de 18 anos; e uma para profissionais e gestores de saúde (2), sendo as duas últimas autoaplicáveis.

Frente à grande importância da APS dentro dos sistemas de saúde e dada a possibilidade de avaliar sua qualidade através da PCATool, visando a um planejamento em saúde mais bem qualificado e que imponha ao poder público um uso mais eficiente de recursos, esta revisão sistemática se propôs a sumarizar as evidências disponíveis sobre a extensão da integralidade na APS, considerando os serviços disponíveis e prestados aos usuários em diferentes países.

\section{MATERIAIS E MÉTODOS}

Realizou-se uma revisão sistemática a partir da seguinte questão norteadora: "Qual é a presença e extensão do atributo integralidade na APS segundo a aplicação da ferramenta Primary Care Assessment Tool (PCATool)?". O protocolo de estudo da pesquisa está disponível no registro International Prospective Register of Systematic Reviews (PROSPERO) (3) sob o número CRD42020202256.

Foram incluídos: estudos observacionais que avaliassem o atributo "integralidade" na APS através da ferramenta PCATool versão usuários; que incluíssem participantes com idade igual ou superior a 18 anos; e que tivessem sido publicados de $1^{\circ}$ de janeiro de 2017 a $1^{\circ}$ de abril de 2019 , período que enfoca as publicações mais recentes na área, sem restrições de idioma ou país. Na PCATool, a integralidade é avaliada em dois aspectos, serviços prestados (efetivamente executados na APS) e serviços disponíveis (disponíveis através da APS, inclusive por encaminhamento a níveis mais complexos de atenção).

Os itens da PCATool são respondidos utilizando uma escala Likert, com valores de 1 a 4 para cada resposta. Posteriormente, os valores obtidos são utilizados para calcular um escore médio de cada componente ou atributo da APS. Ainda é possível calcular o Escore Essencial da APS, com valores mais altos sugerindo maior presença e extensão dos atributos essenciais nos serviços. Tanto os escores dos componentes ou atributos quanto o Escore Essencial podem ser transformados em valores de 0 a 10, visando a facilitar a leitura dos resultados. Embora a definição de integralidade e a avaliação dos atributos não apresente uma grande variação entre os países, diferentes países podem adotar pontos de corte diferentes. No Brasil, um escore de $\geq 6,6$ (alto) indica orientação à APS, enquanto o escore $<6,6$ (baixo) indica presença e extensão menor dos atributos da APS (4).

Foram realizadas buscas nas bases de dados PubMed, Biblioteca Virtual em Saúde (BVS) e Scopus, no período de maio a junho de 2019. Para a base PubMed, utilizou-se a seguinte estratégia de busca: ((PCATool[All Fields] OR "Primary Care Assessment
Tool" [All Fields])) AND "Primary Health Care" [Mesh]. A estratégia utilizada nas bases BVS e Scopus foi: PCATool OR "Primary Care Assessment Tool" AND "Primary Health Care".

Os processos de busca, triagem e determinação de elegibilidade foram realizados de forma independente por duas autoras (JRT e GMG). Caso houvesse discordância, uma terceira revisora (DBV) era consultada. As revisoras foram treinadas previamente com o objetivo de padronizar o processo de busca dos artigos nas diversas bases de dados.

A escala de Newcastle-Ottawa (5) foi utilizada para avaliar a qualidade dos estudos observacionais incluídos nesta revisão. A escala avalia os estudos com base em critérios relativos à seleção e comparabilidade entre coortes e em critérios relacionados aos desfechos de estudo. Para os estudos transversais foi utilizada uma lista adaptada com cinco aspectos da escala Newcastle-Ottawa (5), com objetivo de avaliar o risco de viés com base em representatividade da amostra, definição da exposição, definição da condição de apresentação, taxa de resposta e determinação do resultado.

Os seguintes dados foram extraídos e registrados em uma planilha de análise: referência (autor e ano de publicação), local do estudo, características dos participantes (número e idade), grau de orientação (escore) para integralidade quanto aos serviços disponíveis, grau de orientação (escore) para integralidade quanto aos serviços prestados, resultados e qualidade dos estudos segundo a escala de Newcastle-Ottawa (5).

\section{RESULTADOS}

Foram incluídos 13 artigos que avaliaram a integralidade na APS em diferentes países utilizando a PCATool. A busca inicial nas bases de dados resultou em 124 artigos, sendo 24 na PubMed, 63 na BVS e 37 no Scopus. Desse total, foram excluídas 70 duplicatas entre as bases de dados, restando, assim, 54 artigos para avaliação. Essa etapa resultou em 15 artigos selecionados para leitura na íntegra; após a leitura, 13 artigos foram incluídos na pesquisa. Os motivos das exclusões estão descritos no fluxograma de seleção de estudos (figura 1).

Após leitura completa dos 13 artigos selecionados, foi realizada a avaliação da qualidade dos estudos segundo a escala de Newcastle-Ottawa (5), sendo atribuídas pontuações de 4 a 8 para cada estudo (tabela 1). Sendo assim, os 13 estudos selecionados (6-18) se encaixam dentro da qualidade esperada para esta pesquisa. Os dados obtidos dos estudos foram inseridos em um quadro de extração de dados pré-definido (tabela 2).

Dos 13 artigos selecionados, sete foram publicados no ano de 2017, cinco em 2018 e um em 2019. Oito estudos foram realizadas no continente asiático (quatro na China, dois no Japão e dois no Vietnã). No continente americano, os quatro estudos incluídos foram do Brasil. O continente africano ficou em terceiro lugar, com apenas um estudo, realizado no Malawi.

Doze estudos foram realizados em apenas um momento; apenas um estudo relatou a aplicação da PCATool em dois momentos no mesmo local, mas com participantes diferentes. Em relação ao tipo de local estudado, cinco estudos investigaram centros de saúde comunitários e quatro investigaram unidades básicas de saúde/Estratégia Saúde da Família (UBS/ ESF). Os demais estudos foram realizados em centro de saúde pública, rede de prática de cuidados primários, clínicas de aldeia e local de cuidado (um estudo em cada local). Quanto ao tipo de população estudada, 10 estudos incluíram adultos 
FIGURA 1. Fluxograma de seleção dos estudos incluídos na pesquisa, 2019
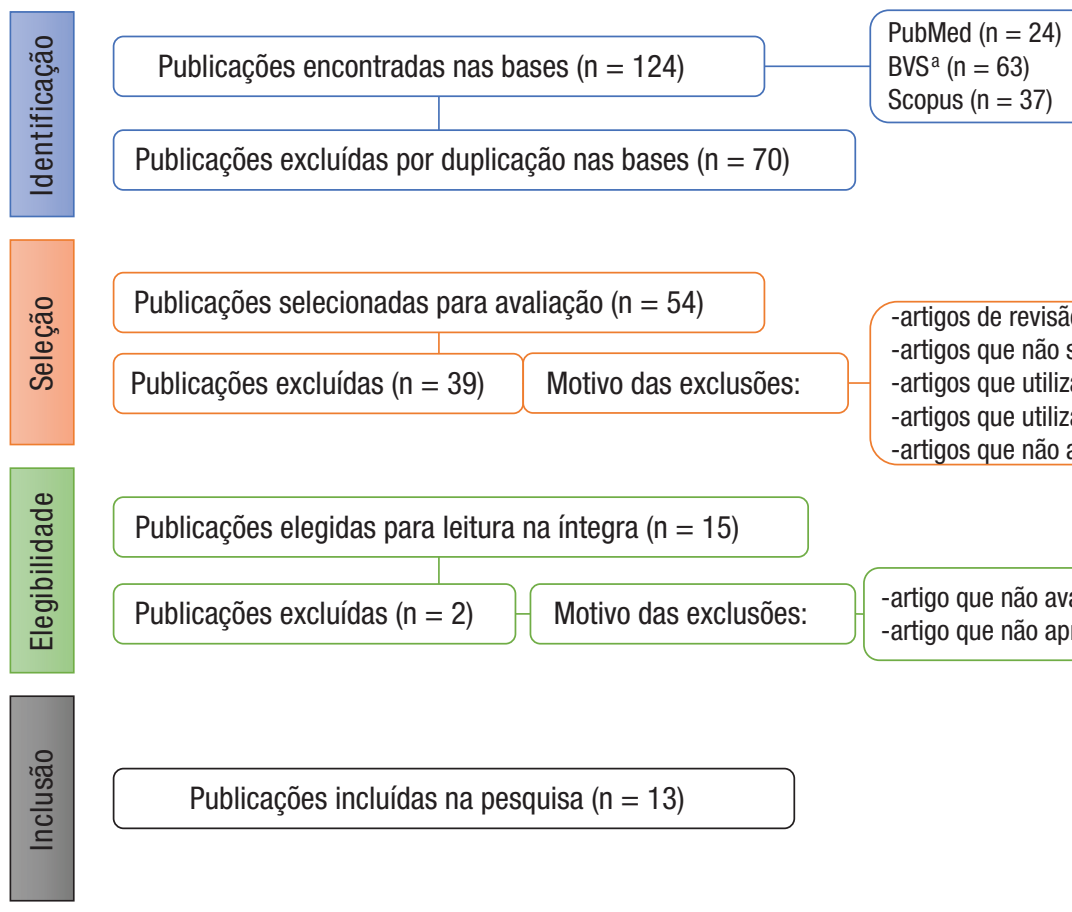

a BVS: Biblioteca Virtual em Saúde.

artigo que não avaliou 0 atributo da integralidade $(n=1)$

-artigo que não apresentou amostra somente de adultos $(n=1)$

TABELA 1. Avaliação de viés conforme Escala de Newcastle-Ottawa

\begin{tabular}{|c|c|c|c|c|c|c|c|c|c|}
\hline & $\begin{array}{l}\text { Amostragem } \\
\text { aleatória }\end{array}$ & $\begin{array}{l}\text { Amostragem } \\
\text { imparcial }\end{array}$ & $\begin{array}{l}\text { Amostra com } \\
\text { sujeitos bem } \\
\text { descritos }\end{array}$ & $\begin{array}{l}\text { Tamanho da } \\
\text { amostra }\end{array}$ & $\begin{array}{l}\text { Utilização } \\
\text { PCATool }\end{array}$ & $\begin{array}{l}\text { Avaliadores } \\
\text { imparciais }\end{array}$ & $\begin{array}{l}\text { Taxa de } \\
\text { resposta }\end{array}$ & $\begin{array}{c}\text { Tipo de teste estatístico } \\
\text { utilizado }\end{array}$ & Total \\
\hline Wei et al. (6) & 1 & 0 & 1 & 1 & 1 & 1 & 0 & 0 & 5 \\
\hline Gontijo et al. (8) & 1 & 1 & 1 & 1 & 0 & 1 & 1 & 0 & 6 \\
\hline Pinto et al. (9) & 1 & 1 & 1 & 1 & 1 & 1 & 0 & 1 & 7 \\
\hline Dullie et al. (10) & 1 & 1 & 1 & 1 & 0 & 1 & 0 & 1 & 6 \\
\hline Zhong et al. (12) & 0 & 0 & 1 & 1 & 1 & 0 & 0 & 1 & 4 \\
\hline Silva et al. (13) & 1 & 1 & 1 & 1 & 1 & 0 & 0 & 1 & 6 \\
\hline Hoa et al. (14) & 0 & 0 & 1 & 1 & 1 & 0 & 0 & 1 & 4 \\
\hline Li et al. (15) & 1 & 0 & 1 & 1 & 1 & 1 & 1 & 1 & 7 \\
\hline Aoki e Inoue (16) & 1 & 1 & 1 & 1 & 0 & 0 & 0 & 1 & 5 \\
\hline Aoki et al. (17) & 1 & 1 & 1 & 1 & 0 & 1 & 0 & 1 & 5 \\
\hline
\end{tabular}
Critérios para a avaliação de estudos transversais (máximo de 8 pontos): amostragem aleatória: 1 - sim, 0 - não; amostragem imparcial: 1 - sim, 0 - não; amostra com sujeitos bem descritos: 1 - sim, 0 - não; tamanho da amostra:
1 - maior ou igual a 300 sujeitos, 0 - menor que 300 sujeitos; utilização de PCAT: 1 - Versão padrão PCATool, 0 - Versão adaptada PCATool; avaliadores imparciais: 1 - sim, 0 - não; taxa de resposta: 1 - maior ou igual a $70 \%, 0$ - menor que $70 \%$; tipo de teste estatístico: 1 - teste T, 0 - outro teste estatístico.

( $\geq 18$ anos), seguidos de idosos hipertensos, homens adultos e adultos cuidadores de crianças menores de 12 anos, sendo as últimas três categorias representadas por um estudo cada.

Dez estudos utilizaram a PCATool com sua respectiva adaptação e validação para o país de forma completa; apenas um estudo, no Brasil, utilizou uma versão reduzida da ferramenta. Em relação ao local de aplicação da ferramenta, ocorreu na unidade de saúde avaliada em sete estudos; na casa do participante em quatro estudos; e por meio tecnológico (telefone ou e-mail) em dois estudos. Apenas quatro estudos abordaram as características dos entrevistadores, compostos por acadêmicos de medicina, gestão em saúde e pós-graduação e por agentes comunitários de saúde, todos devidamente treinados quanto à aplicação da ferramenta.

A avaliação da integralidade quanto à disponibilidade dos serviços classificou oito locais como de baixo grau de orientação à APS, enquanto cinco apresentaram alto grau de orientação. Já para os serviços prestados, nove locais avaliados foram 
TABELA 2. Síntese das publicações incluídas no estudo, 2019

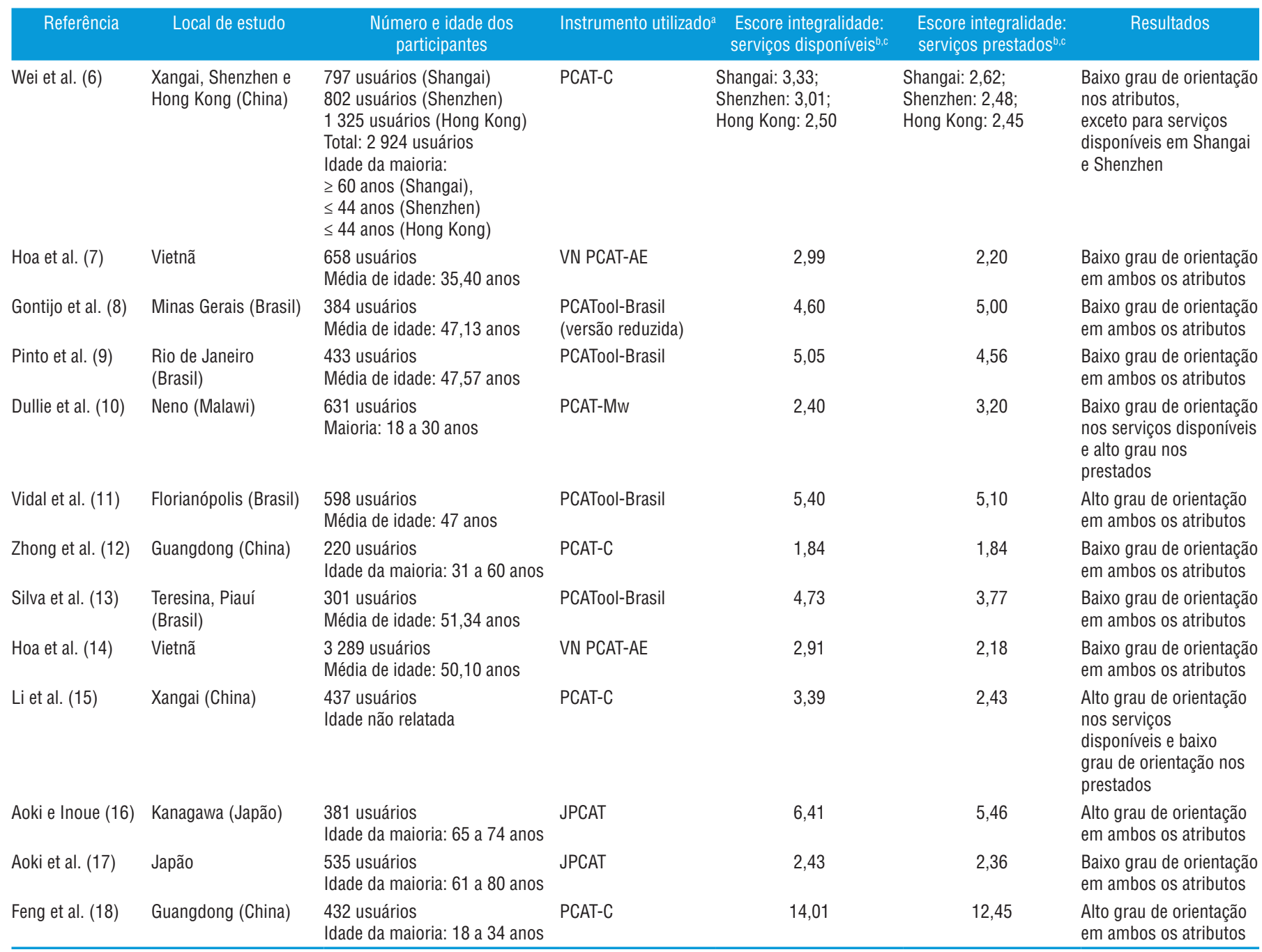

PCATool: Primary Care Assessment Tool

:

' Referências da PCATool China (PCAT-C), Japão (JPCAT), Vietnã (VN PCAT-AE) e Malawi (PCAT-Mw): escore < 3: baixo grau de orientação; $\geq 3$ : alto grau de orientação

classificados como de baixo grau de orientação, e apenas quatro apresentaram alto grau de orientação à APS.

\section{DISCUSSÃO}

A análise dos estudos incluídos nesta pesquisa mostrou que a maioria dos locais avaliados apresentou nível inadequado de orientação à APS no atributo integralidade, tanto nos serviços disponíveis quanto nos serviços prestados. As questões que compreendem o atributo da integralidade englobam tanto demandas relacionadas ao processo saúde-doença quanto a ações de prevenção, promoção e reabilitação da saúde $(1,19)$.

O baixo grau de orientação à APS nos serviços disponíveis e prestados pode ser interpretado como a não compreensão das reais demandas dos usuários atendidos pelos respectivos centros de saúde. É necessário que esses locais, através de seus gestores e trabalhadores de saúde, reconheçam as necessidades e prioridades dos usuários e viabilizem recursos humanos e estruturais para o seu completo atendimento (19). Dessa maneira, são necessárias intervenções de toda a rede de saúde para lidar com os problemas apresentados pela APS (20).

Alguns estudos incluídos nesta revisão foram realizados em países onde a APS ainda convive com a atenção centrada no modelo hospitalar, ou seja, focada no sistema terciário, enfraquecendo, assim, o primeiro nível de assistência à saúde. $\mathrm{O}$ fortalecimento da APS é, de certo modo, recente, mas tem-se mostrado como a principal base para a consolidação do sistema de saúde (19). É necessário melhorar a cartela de serviços disponíveis e constituir novos arranjos no quadro de colaboradores dos centros de saúde. A interação e o fortalecimento do contato com outros pontos da rede de cuidados de saúde são de extrema importância, pois essa rede pode e deve complementar os serviços que não estão disponíveis na APS (11).

Para entender as evidências sobre o atributo integralidade, é necessário que a organização do sistema de saúde dos países aqui citados seja considerada. A maioria dos estudos elencados foi realizada na China. Esse fato pode ser explicado pelas mudanças recentes que o país tem realizado para reformular a 
APS. No ano de 2009, foi apresentada uma reforma nacional de saúde com a finalidade d e fornecer s erviços de A PS s eguros, acessíveis e eficazes para a população chinesa. A reforma englobou serviços médicos básicos e fortaleceu o sistema (que até então era centrado nos níveis terciários) no nível de atenção primária (21). Assim, o país fez investimentos grandiosos na APS para atender ao objetivo da reforma nacional de saúde, tendo como destaque um acesso ao sistema mais equitativo e com maior acessibilidade para os cidadãos (22). A China esperava alcançar a cobertura universal de saúde até o ano de 2020 (23).

Outro país que teve destaque em termos do número de estudos apresentados foi o Brasil. Esse dado pode ser sustentado pela mudança que ocorreu no sistema de saúde brasileiro com a adesão prioritária da ESF, promovendo a ampliação e o fortalecimento da APS no país. Com essa nova estratégia de APS, o processo de trabalho obteve uma reorientação, intensificando a resolubilidade nos processos de saúde da população atendida e auxiliando os fundamentos e as diretrizes da APS (24).

É papel fundamental do trabalhador em saúde, que atua na frente de apoio e cuidado ao usuário, desenvolver uma sensibilidade que permita vislumbrar o paciente como uma pessoa completa, levando em consideração não apenas a sua história clínica, mas a sua cultura, o meio em que vive e as relações sociais que desenvolveu ao longo de sua trajetória de vida (25). Um fator de destaque é a importância que essa temática deve possuir na formação e educação continuada dos profissionais de saúde e de apoio que atuam nesses locais de prestação de serviço.

A PCATool permite avaliar a presença e a extensão dos atributos essenciais e derivados encontrados na APS (1). A avaliação dos centros de saúde através dessa ferramenta é fundamental para a melhoria dos processos e das ações em saúde (19). O emprego de instrumentos validados para avaliar os serviços de saúde na APS é de extrema importância, e o uso da PCATool tem aumentado a cada dia (26). Vários países (27), como Brasil, Canadá, Espanha e China, têm realizado a adaptação e validação da ferramenta para adequação e utilização em seus respectivos territórios.

A PCATool original foi idealizada nos Estados Unidos (2), em 2001, e conta com 92 itens, sendo 37 questões no eixo da integralidade. Já no Brasil, quando a ferramenta foi adaptada e validada (PCATool-Brasil) por Harzheim et al. em 2013 (28), passou a ter 87 itens em sua totalidade e 35 itens no atributo da integralidade. Foi utilizada em sua forma completa nos estudos de Pinto et al. (9), Vidal et al. (11) e Silva et al. (13). Já a versão reduzida da PCATool-Brasil foi criada por Oliveira et al. (29) em 2013, contando com 23 questões no total e apenas sete questões voltadas à integralidade. Essa versão foi utilizada no estudo de Gontijo et al. (8).

A ferramenta adaptada e validada para o Japão (JPCAT) foi idealizada em 2016 (30) e possui 29 questões, sendo 10 sobre integralidade. A JPCAT foi utilizada nos estudos de Aoki e Inoue (16) e Aoki et al. (17). No Vietnã, a ferramenta foi adaptada e validada (VN PCAT-AE) por Hoa et al. (14) em 2018, contendo 70 questões, sendo 36 referentes à integralidade. Além de ter sido utilizada no estudo mencionado, também foi aplicada na pesquisa de Hoa et al. (7), em 2019.

O estudo de Dullie et al. (20) utilizou a ferramenta adaptada e validada para o Malawi (PCAT-Mw) (10), que conta com 29 questões, sendo 12 sobre integralidade. Nos estudos de Wei et al. (6), Zhong et al. (12), Li et al. (15) e Feng et al. (18), realizados na China, onde existem várias versões da PCATool e as questões relacionadas à integralidade variam em cada estudo (31), foi utilizada a ferramenta PCAT-C, adaptada e validada para a China por Yang et al. (32) em 2013. Essa versão conta com 63 questões, das quais oito compõem o eixo da integralidade.

A adaptação do instrumento PCATool segundo as características e peculiaridades de cada país demonstra a grande importância de uma avaliação efetiva e de boa qualidade da APS. No entanto, as diferenças entre as versões da ferramenta no tocante aos itens que compõem a integralidade podem gerar inconsistência na padronização e comparação entre as pesquisas que envolvem as PCATool de cada nacionalidade, fator que dificulta a conferência das questões nas pesquisas entre os países.

Destaca-se como ponto forte deste estudo a explanação do uso da PCATool no mundo, que envolveu a comparação e sistematização da maneira como o instrumento é utilizado por cada país. Outro fator importante é a compreensão de como os países estão organizando e realizando melhorias constantes em seus sistemas de saúde e nas equipes de profissionais e gestores a partir dos resultados apresentados nos estudos envolvendo a PCATool.

Este estudo apresenta como limitação as diferenças entre as versões da PCATool em cada país. Embora tenham sido utilizadas versões traduzidas, adaptadas e validadas para cada países, essas versões ainda apresentam inconsistências no eixo da integralidade, podendo causar divergências no entendimento dos participantes e em suas respectivas respostas. Como pontos fortes, o rigor metodológico foi seguido e todas as etapas pertinentes a um estudo de revisão sistemática foram cumpridas. Outra limitação que podemos citar é o curto período de busca (2 anos). Entretanto, o objetivo foi revisar o cenário mais atualizado quanto à integralidade.

Por meio desta revisão sistemática, foi possível identificar que o grau de orientação à APS no atributo integralidade foi insatisfatório na maioria dos países investigados, tanto no subgrupo dos serviços disponíveis quanto nos serviços prestados. Diante desse resultado, destaca-se a importância da frequente mensuração da qualidade dos serviços de saúde na APS a partir da visão dos usuários e o aumento e a flexibilização dos serviços disponíveis na APS para que, assim, seja possível atender a todas as demandas de usuários ou, de outra forma, encaminhálos para outro ponto da rede de atenção à saúde, fortalecendo, assim, o contato com outros pontos da rede. Para o fortalecimento dos serviços prestados, recomenda-se que as instituições de saúde e seus respectivos gestores prestem maior apoio à melhoria das estruturas físicas, tecnológicas e, sobretudo, humanas, com capacitações e treinamentos para os profissionais de saúde por meio de processos de educação permanente.

Contribuições dos autores. JRT e DBV conceberam o estudo. JRT e GMG coletaram os dados e analisaram os dados e contribuíram com dados e ferramentas de análise. JRT escreveu a primeira versão do artigo. DBV revisou o artigo e supervisionou todo o estudo. Todos os autores revisaram e aprovaram a versão final.

\section{Conflitos de interesse. Nada declarado pelos autores.}

Declaração. As opiniões expressas no manuscrito são de responsabilidade exclusiva dos autores e não refletem necessariamente a opinião ou política da RPSP/PAJPH ou da Organização Pan-Americana da Saúde (OPAS). 


\section{REFERÊNCIAS}

1. Starfield B. Atenção primária: equilíbrio entre necessidades de saúde, serviços e tecnologia. Brasília: UNESCO, Ministério da Saúde; 2002.

2. Shi L, Starfield B, Xu J. Validating the Adult Primary Care Assessment Tool. J Fam Pract. 2001;50(2):161-75.

3. National Institute for Health Research. Prospero - International prospective register of systematic reviews. 2019. Disponível em: https://www.crd.york.ac.uk/prospero/ Acessado em 25 de maio de 2021.

4. Brasil, Ministério da Saúde, Secretaria de Atenção Primária à Saúde, Departamento de Saúde da Família. PCATool/Brasil 2020. Manual do instrumento de avaliação da atenção primária à saúde. Brasília: Ministério da Saúde; 2020. Disponível em: https:/ /www.ufrgs.br/ telessauders/documentos/20200506_Pcatool_versao_Final.pdf.pdf Acessado em novembro de 2021

5. Wells G, Shea B, O'Connell D, Peterson J, Welch V, Losos M, et al. The Newcastle-Ottawa Scale (NOS) for assessing the quality of nonrandomised studies in meta-analyses. 2011. Disponível em: http:/ / www.ohri.ca/programs/clinical_epidemiology/oxford.asp Acessado em 24 de maio de 2021.

6. Wei X, Yin J, Wong SYS, Griffiths SM, Zou G, Shi L. Private ownership of primary care providers associated with patient perceived quality of care: a comparative cross-sectional survey in three big Chinese cities. Medicine (Baltimore). 2017;96(1):e5755. doi: 10.1097/ MD.0000000000005755

7. Hoa NT, Tam NM, Derese A, Markuns JF, Peersman W. Patient experiences of primary care quality amongst different types of health care facilities in central Vietnam. BMC Heal Serv Res. 2019;19(1):275. doi: 10.1186/s12913-019-4089-y

8. Gontijo TL, Duarte AGS, Guimarães EAA, Silva J. Avaliação da atenção primária: o ponto de vista de usuários. Saúde Debate. 2017;41(114):741-52. doi: 10.1590/0103-1104201711406

9. Pinto LF, HarzheimE, Hauser L, D'Avila OP, Gonçalves MR, Travassos $P$, et al. A qualidade da Atenção Primária à Saúde na Rocinha Rio de Janeiro, Brasil, na perspectiva dos cuidadores de crianças e dos usuários adultos. Cienc Saude Colet. 2017;22(3):771-81. doi: 10.1590/1413-81232017223.33132016

10. Dullie L, Meland E, Hetlevik $\varnothing$, Mildestvedt T, Gjesdal S. Development and validation of a Malawian version of the primary care assessment tool. BMC Fam Pr. 2018;19(1):63. doi: 10.1186/ s12875-018-0763-0

11. Vidal TB, Tesser CD, Harzheim E, Fontanive PVN. Avaliação do desempenho da Atenção Primária à Saúde em Florianópolis, Santa Catarina, 2012: estudo transversal de base populacional. Epidemiol Serv Saude. 2018;27(4):e2017504. doi: 10.5123/S1679-49742018000400006

12. Zhong C, Kuang L, Li L, Liang Y, Mei J, Li L. Equity in patient experiences of primary care in community health centers using Primary Care Assessment Tool: a comparison of rural-to-urban migrants and urban locals in Guangdong, China. Int J Equity Heal. 2018;17(1):51. doi: 10.1186/s12939-018-0758-4

13. Silva AN, Silva SA, Silva ARV, Araújo TME, Rebouças CBA, Nogueira LT. A avaliação da Atenção Primária a Saúde na perspectiva da população masculina. Rev Bras Enferm. 2018;71(2):255-63. doi: 10.1590/0034-7167-2016-0651

14. Hoa NT, Tam NM, Peersman W, Derese A, Markuns JF. Development and validation of the Vietnamese Primary Care Assessment Tool. PLoS One. 2018;13(1):e0191181. doi: 10.1371/journal.pone.0191181

15. Li H, Wei X, Wong MCS. Changes of the perceived quality of care for older patients with hypertension by community health centers in Shanghai. BMC Fam Pr. 2017;18(1):114. doi: 10.1186/ s12875-017-0683-4

16. Aoki T, Inoue M. Association between health literacy and patient experience of primary care attributes: a cross-sectional study in Japan. PLoS One. 2017;12(9):e0184565. doi: 10.1371/journal. pone. 0184565
17. Aoki T, Miyashita J, Yamamoto Y, Ikenoue T, Kise M, Fujinuma Y, et al. Patient experience of primary care and advance care planning: a multicentre cross-sectional study in Japan. Fam Pr. 2017;34(2):20612. doi: $10.1093 /$ fampra/cmw126

18. Feng S, Shi L, Zeng J, Chen W, Ling L. Comparison of primary care experiences in village clinics with different ownership models in guangdong province, China. PLoS One. 2017;12(1):e0169241. doi: 10.1371/journal.pone.0169241

19. Mesquita Filho M, Luz BSR, Araújo CS. A Atenção Primária à Saúde e seus atributos: a situação das crianças menores de dois anos segundo suas cuidadoras. Cienc Saude Colet. 2014;19(7):2033-46. doi: 10.1590/1413-81232014197.17322013

20. Dullie L, Meland E, Mildestvedt T, Hetlevik $\varnothing$, Gjesdal S. Quality of primary care from patients' perspective: A cross sectional study of outpatients' experience in public health facilities in rural Malawi. BMC Heal Serv Res Res. 2018;18(1):872. doi: 10.1186/ s12913-018-3701-x

21. China. Opiniões do Conselho sobre o aprofundamento da reforma dos cuidados de saúde. 2009. Disponível em: http:/ / www.gov.cn/ jrzg/2009-04/06/content_1278721.htm Acessado em 25 de maio de 2021.

22. Li X, Lu J, Hu S, Cheng KK, De Maeseneer J, Meng Q, et al. The primary health-care system in China. Lancet. 2017;390(10112):2584-94. doi: 10.1016/S0140-6736(17)33109-4

23. Meng Q, Mills A, Wang L, Han Q. What can we learn from China's health system reform? BMJ. 2019;365:12349. doi: 10.1136/bmj.12349

24. Brasil, Ministério da Saúde, Secretaria de Atenção à Saúde, Departamento de Atenção Básica. Política Nacional de Atenção Básica. Brasília: Ministério da Saúde; 2012. Disponível em: http:/ / 189.28.128.100/dab/docs/publicacoes/geral/pnab.pdf Acessado em 04 de outubro de 2021.

25. Paula CC, Silva CB, Tassinari TT, Padoin SMDM. Factors that affect first contact access in the primary health care: integrative review. J Res Fundam Care Online. 2016;8(1):4056-78. doi: 10.9789/21755361.2016.v8i1.4056-4078

26. Stein AT. A avaliação dos serviços de saúde deve ser realizada com instrumentos validados. Epidemiol Serv Saude. 2013;22(1):179-81. doi: $10.5123 /$ S1679-49742013000100019

27. D'Avila OP, Pinto LFS, Hauser L, Gonçalves MR, Harzheim E. O uso do Primary Care Assessment Tool (PCAT): uma revisão integrativa e proposta de atualização. Cienc Saude Colet. 2017;22(3):855-65. doi: 10.1590/1413-81232017223.03312016

28. Harzheim E, Oliveira MMC, Agostinho MR, Hauser L, Stein AT, Gonçalves MR, et al. Validação do instrumento de avaliação da atenção primária à saúde: PCATool-Brasil adultos. Rev Bras Med Fam Comunidade. 2013;8(29):274-84. doi: 10.5712/rbmfc8(29)829.

29. Oliveira MMC, Harzheim E, Riboldi J, Duncan BB. PCATool-ADULTO-BRASIL: uma versão reduzida. Rev Bras Med Fam Comunidade. 2013;8(29):256-63. doi: 10.5712/rbmfc8(29)823

30. Aoki T, Inoue M, Nakayama T. Development and validation of the Japanese version of Primary Care Assessment Tool. Fam Pract. 2016;33(1):112-7. doi: 10.1093/fampra/cmv087

31. Wang W, Haggerty J, Loban EK, Liu X. Evaluating primary health care performance from user perspective in china: Review of survey instruments and implementation issues. Int J Environ Res Public Health. 2019;16(6):926. doi: 10.3390/ijerph16060926

32. Yang H, Shi L, Lebrun LA, Zhou X, Liu J, Wang H. Development of the Chinese Primary Care Assessment Tool: data quality and measurement properties. Int J Qual Health Care. 2013;25(1):92-105. doi: 10.1093/intqhe/mzs072

Manuscrito submetido em 7 de agosto de 2021. Aceito em versão revisada em 22 de setembro de 2021. 


\section{Measuring comprehensiveness of care using the Primary Care Assessment Tool: systematic review}

ABSTRACT Objective. To assess the comprehensiveness of primary healthcare (PHC) in different countries.

Method. PubMed, Virtual Health Library (BVS), and Scopus were systematically searched. Observational studies published from 2017 to 2019, using the Adult Primary Care Assessment Tool (PCAT) to assess comprehensiveness were included without limits regarding language of publication or country. The quality of studies was assessed using the Newcastle-Ottawa scale (NOS).

Results. Of 124 articles initially selected, 13 were included: four from China, two from Japan, and two from Vietnam; considering the Americas, all four studies were performed in Brazil. Only one study from Africa, performed in Malawi, was included. The quality of studies according to the NOS was acceptable. Considering the availability of services, eight facilities had low comprehensiveness, vs. five with high comprehensiveness. Considering the services performed at the facility, nine had low comprehensiveness, and only four had high comprehensiveness.

Conclusion. The low degree of PHC orientation in terms of comprehensiveness in terms of both services performed and services provided may reflect a lack of understanding of the demands of users, and indicates the need for concrete action to strengthen PHC as the basis of healthcare systems.

Keywords $\quad$ Primary health care; health care evaluation mechanisms; integrality in health.

\section{Evaluación de la integralidad en la atención primaria de salud por medio de la herramienta de evaluación de la atención primaria: revisión sistemática}

RESUMEN Objetivo. Evaluar la integralidad en la atención primaria de salud en distintos países.

Métodos. Se realizó una revisión sistemática en las bases de datos de PubMed, la Biblioteca Virtual de Salud y Scopus. Se seleccionaron estudios observacionales publicados en el período del 2017 al 2019, sin restricciones de idioma ni país, en los cuales se evaluara la integralidad en la atención primaria de salud por medio de la herramienta de evaluación de la atención primaria de salud (Primary Care Assessment Tool o PCATool), en su versión para adultos. La calidad de los estudios se determinó con la escala de Newcastle-Ottawa.

Resultados. En el estudio se incluyeron 13 de los 124 artículos seleccionados inicialmente, a saber, cuatro de China, dos de Japón y cuatro de Vietnam; además de cuatro estudios del continente americano realizados en Brasil y un único estudio correspondiente a África hecho en Malawi. La calidad de los estudios determinada con la escala de Newcastle-Ottawa fue satisfactoria. En la evaluación de la integralidad en cuanto a la disponibilidad de los servicios se clasificaron ocho lugares con un grado bajo y cinco con un grado alto de orientación hacia la atención primaria de salud. En lo que respecta a los servicios prestados, nueve lugares se clasificaron con un grado bajo y cuatro con un grado alto de orientación hacia la atención primaria de salud. Conclusión. El grado bajo de orientación hacia la atención primaria de salud en cuanto a la integralidad de los servicios disponibles y prestados puede interpretarse como una falta de comprensión de las demandas reales de los usuarios, lo cual exige una acción concreta para fortalecer la atención primaria de salud como base de los sistemas de salud.

Palabras clave Atención primaria de salud; mecanismos de evaluación de la atención de salud; integralidad en salud. 